\title{
Research and analysis on the current quality of shower products
}

\author{
Shanyu Liu ${ }^{1}$, Kejing Li $^{1 *}$, Kaifang Wang ${ }^{1}$, Chen Wang ${ }^{1}$ \\ ${ }^{1}$ China Building Material Test \& Certification Group (Shaanxi) Co. Ltd, Xi'an, Shaanxi, 710049, China
}

\begin{abstract}
Shower head, also known as shower head, is a device that allows water to diverge and flow out in the form of droplets or sprays. It was originally a device for watering flowers, potted plants and other plants. Later, someone modified it to be used as a shower, making it a common device in the bathroom, called a shower head, mainly used for showering in households or public places. In terms of structure, the shower is generally composed of a body, a switching component, a water distribution component, a rotor, a water outlet, a face cover, a water outlet component, a water-saving device, a plug, a button, and so on. This article mainly analyzes the industrial distribution of shower heads, product quality spot checks and the causes of quality problems.
\end{abstract}

\section{Introduction}

In recent years, as my country's economy continues to grow rapidly, the domestic hardware and bathroom industry has achieved unprecedented development[1]. Among them, the production scale and capacity of shower shower products rank first in the world, and the product quality has also been greatly improved. At present, the annual output of domestic shower products has exceeded two, and there are about 200 manufacturers, mainly in South China, East China, North China, and Southwest China. Among them, southern China is the region with the largest distribution of domestic shower shower products, mainly in Foshan, Guangdong, Jiangmen, Guangdong, Heshan, Guangdong; shower showers in East China are mainly produced in Quanzhou, Fujian and Xiamen, Wenzhou, Zhejiang, Yuhuan, Zhejiang, Shanghai and other regions dominate; North China and Southwest China are dominated by cities such as Beijing, Tianjin, Hebei Tangshan, and Chongqing[2]. The number of enterprises and output in these areas are relatively small compared with those in South and East China. At present, the domestic shower head sales market is mainly divided into seven regions in North China, East China, South China, Southwest China, Central China, Northeast China, and Northwest China. Among them, North China, East China and South China are mature markets, mainly concentrated in more developed first- and second-tier cities and Developed regions. The Southwest, Central China, Northeast, and Northwest regions are relatively lowprofile markets. Because of their low maturity, there is more room for development, and they are also markets for mid-to-high-end brands in the future[3].

\section{Analysis of product quality spot check}

From 2015 to 2019, the State Administration for Market Regulation organized five consecutive national quarterly inspections on shower products. The situation is as follows: in 2015, a total of Beijing, Liaoning, Shanghai, Jiangsu, Zhejiang, Fujian, Guangdong 72 kinds of shower products produced by 72 enterprises in 7 provinces and municipalities directly under the Central Government, the coverage rate of random inspection was $36 \%$, and the qualified rate of random inspection products was $84.7 \%$; in 2016, a total of random inspections of Beijing, Liaoning, Shanghai, Jiangsu, Zhejiang, Fujian, 90 kinds of shower products produced by 79 companies including Guangdong, the coverage rate of random inspection was $45 \%$, and the qualified rate of random inspection products was $86.7 \%$ [4]; In 2017, a total of 77 shower shower products produced by 77 companies in 3 provinces in Zhejiang, Fujian and Guangdong were inspected, with a coverage rate of $38.5 \%$ and a qualified rate of $77.9 \%$; In 2018, a total of 81 shower products produced by 81 enterprises in 10 provinces and municipalities including Beijing, Hebei, Liaoning, Shanghai, Jiangsu, Zhejiang, Anhui, Fujian, Guangdong, and Sichuan were inspected, and the coverage rate was $40.5 \%$. The product qualification rate was $82.7 \%$; in 2019,89 batches of shower products produced by 89 companies in 7 provinces (cities) including Beijing, Hebei, Jiangsu, Zhejiang, Fujian, Guangdong, Shanghai, etc. were spot-checked, and the spot-check coverage rate was $44.5 \%$, the qualified rate of random inspection products was $88.8 \%$. See Figure 1 for details.

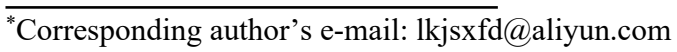




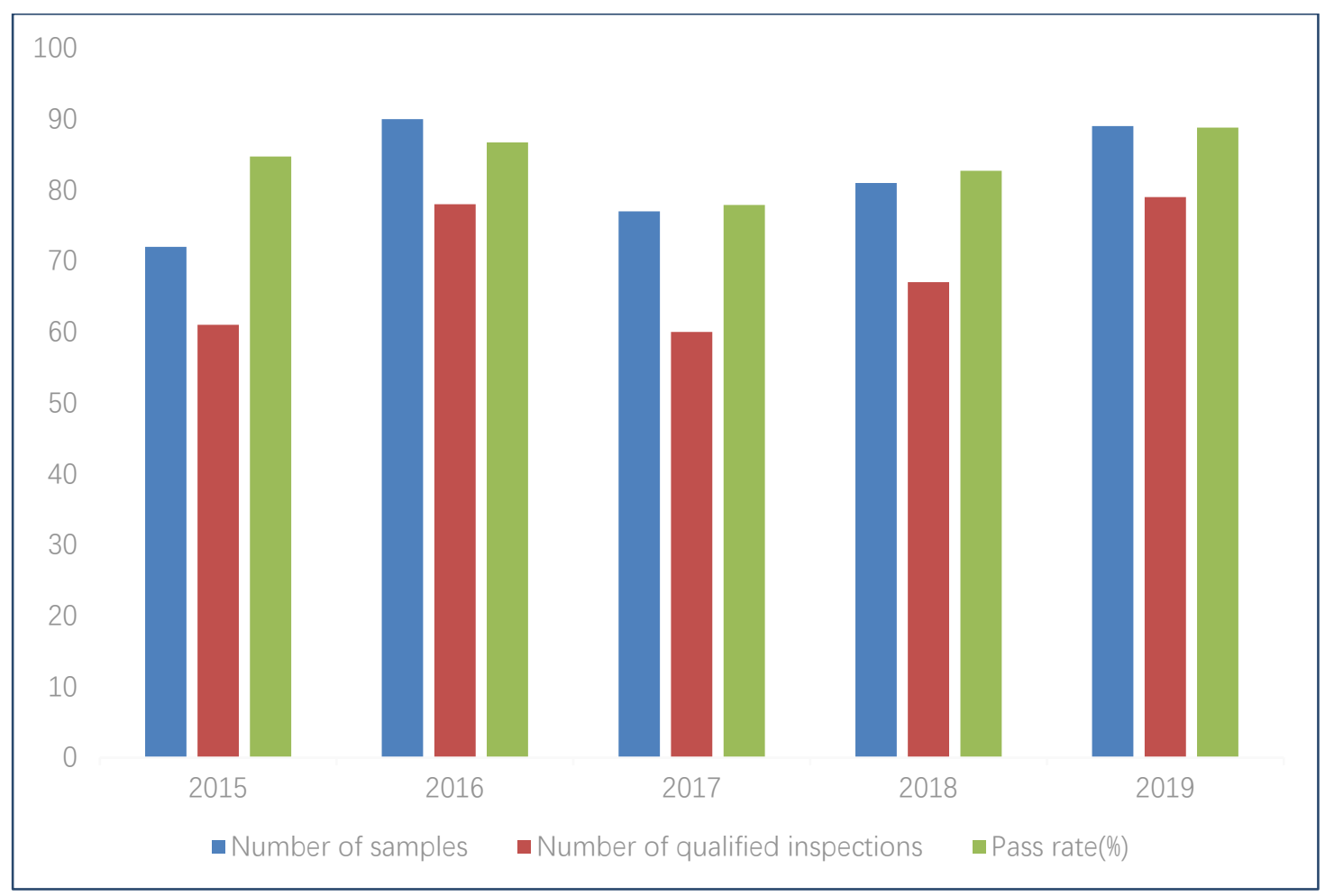

Fig 1. Statistical chart of random inspection of shower head products over the years

\section{Cause analysis of quality problems}

Summarizing the results of the national supervision and random inspection of shower products in the past five years, and analyzing the main problems that exist, most of the manufacturers and unqualified companies are from Fujian, Guangdong and Zhejiang.

Compared with 2018, in 2019, the qualified rate of shower shower products in Fujian Province decreased by 3.1 percentage points[5]. The companies that were spotchecked were mostly located in Xiamen, Nan'an and Quanzhou, and the unqualified companies were all small and micro enterprises; and Compared with 2018, in 2019, the qualification rate of shower shower products in Guangdong Province increased by 6.5 percentage points. The companies that were spot-checked were distributed in Foshan, Kaiping and Heshan, and the unqualified companies were all small and micro enterprises; compared with 2018 In comparison, in 2019, the qualification rate of shower shower products in Zhejiang Province increased by $39 \%$. For Zhejiang Province, the qualification rate of random inspections increased by $39 \%$, which is compared with the sharp decrease in the number of shower products in Zhejiang Province in 2019. Big relationship. The statistics of the number of showers in the three provinces of Fujian, Guangdong and Zhejiang in the past two years are shown in Table 1.

Table1. In the past two years, the number of random inspections of shower products in Fujian, Guangdong and Zhejiang

\begin{tabular}{|c|c|c|c|c|c|}
\hline \multirow{2}{*}{$\begin{array}{c}\text { Serial } \\
\text { number }\end{array}$} & \multirow{2}{*}{ Years } & \multirow{2}{*}{ shower head spot check } & \multicolumn{3}{|c|}{ total batch } \\
\hline & & & Fujian & Guangdong & Zhejiang \\
\hline 1 & 2018 & 81 & 25 & 28 & 18 \\
\hline 2 & 2019 & 89 & 36 & 35 & 8 \\
\hline \multicolumn{3}{|c|}{ Qualification rate changes } & declines & rises & rises \\
\hline
\end{tabular}


Guangdong, Fujian and Zhejiang provinces are major producers of shower shower products. The scale and product quality of the production enterprises vary. There are large enterprises such as Huayi, Jiumu and Shenluda, as well as a large number of small enterprises. Large and medium-sized enterprises have the ability of independent research and development, mold making, parts production, product quality inspection, and product quality is guaranteed; while the molds of small and micro enterprises are not standardized enough to optimize the performance of products; and blindly rely on Pursue profit at low cost, ignoring the impact of the quality of product raw materials on product performance; most small and medium-sized enterprises have poor product quality inspection capabilities, and cannot accurately determine product corrosion resistance, flow rate and other performance, and product technical parameters are determined Inaccurate.

Guangdong Province, Fujian Province and Zhejiang Province, as major export provinces of shower shower products, should play a leading role in the industry, with the goal of producing high-quality, high-performance, environmentally friendly and safe products. In particular, local supervision and management departments and industry associations should play their roles to increase supervision, increase the density of spot checks, and rectify unqualified enterprises.As soon as possible to effectively regulate the shower industry in Guangdong, Fujian and Zhejiang provinces, this is of great significance to the overall development of my country's sanitary ware industry.

\section{Conclusion}

In the future, with the rapid development of modern information technology and manufacturing technology integration and innovation, the building ceramics and sanitary ware industry will develop towards integration, intelligence, and flexibility, and realize the integrated innovation of $\mathrm{R} \& \mathrm{D}$, design, production and management, innovative research and development, Manufacturing and industrial organization methods to realize the flexibility, agility, digitization, networking and coordination of manufacturing and supply chains.

\section{References}

1. UNIDO. Quality Infrastructure[R], 2006.

2. H Katano, K Yokoyama, Y Takei, H Matsuki et al.(2011)The study of the bacterial contamination in the spray water of electronic toilets and in the gluteal and inguinal regions due to splashing following spray water. Microbial Ecology in Health and Disease. vol. 25, pp. 8-14.

3. Ying Huang et al.(2019)A novel multidimensional analysis of writing styles of editorials from China Daily and The New York Times. Lingua. vol. 235, pp. 87-96.

4. KorhanKo et al.(2020)Is Support Vector Regression method suitable for predicting rate of penetration.
Journal of Petroleum Science and Engineering. Vol 194, pp. 19-24.

5. World Bank. The National Quality Infrastructure $[R]$, 2013. 